\title{
Clarificar para Otro: Seis Prácticas Conversacionales Generativas de Cambio Terapéutico desde la Teoría del Cambio Subjetivo
}

\section{Clarifying for an Other: Six Conversational Practices to Foster Therapeutic Change from a Subjective Change Theory Perspective}

\author{
Joaquín Gaete \\ Roberto Arístegui \\ Universidad Adolfo Ibáñez, Chile \\ Mariane Krause \\ Pontificia Universidad Católica de Chile, Chile \\ Rec (17 de Diciembre de 2018) Acept (08 de Julio de 2019)
}

\begin{abstract}
Resumen
Desde la teoría del cambio subjetivo en psicoterapia, este estudio de caso constructor de teoría examina cualitativamente el proceso de cambio de una terapia exitosa. El estudio caracteriza micro-prácticas conversacionales que aparecen en episodios de cambio (EC). Los EC son segmentos de conversación teóricamente vinculados al cambio terapéutico, dado que en ellos aparecen indicadores genéricos de cambio en tres niveles: entrada, proceso, y salida. Se exploraron aquí los 16 episodios conteniendo indicadores genéricos de proceso (nivel 2). Como resultado se describen seis supuestos terapéuticos que facilitaron el logro de autorreferencias preferidas (ARP) en los EC de proceso. Se discute cómo esta caracterización contribuye a comprender la elaboración (subjetiva) de una "teoría" sobre el propio cambio terapéutico como resultado del proceso (intersubjetivo) de clarificar y validar ARP para y con el terapeuta. En este sentido, el estudio permite dar mayor especificidad a la teoría del cambio subjetivo desde el punto de vista del proceso conversacional que con-instituye o legitima el cambio.

Palabras clave: Proceso Psicoterapéutico; Teoría del Cambio Subjetivo; Autorreferencias Preferidas; Estudio de Caso Constructor de Teoría.
\end{abstract}

\begin{abstract}
Drawing from the theory of subjective change in psychotherapy, this theory-building case study examines one successful therapeutic change process. The study characterizes conversational micro-practices featuring in segments of conversation theoretically linked to therapeutic change called change episodes (CE), containing three types of observable generic indicators of change: input, process, and output. For this study the $16 \mathrm{CE}$ containing indicators of the second level (process) were examined. Six conversational practices involving six therapeutic assumptions are presented as a result of the study, which were consequential in discursively accomplishing preferred self-references (PSR) within CE related to process. Given that generating a "subjective theory" about the client's own change process emerges as a byproduct of conversationally clarifying PSR for and with the therapist (i.e., intersubjective validation), this study contributes further specifying the theory of subjective change.

Keywords: Therapeutic process, Theory of Subjective Change, Preferred Self-references; Theory-building case study.
\end{abstract}

Correspondencia: La correspondencia en relación con este artículo debe dirigirse a Joaquín Gaete S., Centro de Estudios y Atención a las Personas (CEAP-V), Los Castaños 41, Viña del Mar, Chile. Email: joaquin.gaete@uai.cl, jgaetes@gmail.com 


\section{Introducción}

La investigación de procesos de cambio tiene como objetivo mejorar la manera en que la conversación en psicoterapia — proceso - facilita el cambio terapéutico (Elliott, 2010). Una estrategia ampliamente utilizada para examinar procesos de cambio ha sido la caracterización de episodios o segmentos de conversación particularmente significativos en su contribución al cambio (e.g., Fernandez et al., 2012). Por ejemplo, Krause, De la Parra, Arístegui, Tomicic, Dagnino \& Echávarri (2006) utilizan el constructo momentos de cambio (MC) para distinguir eventos acotados (e.g., 1 a 3 turnos de palabra), en los que consultante y terapeuta logran un micro-resultado terapéutico. Por otro lado, los episodios de cambio (EC) son segmentos más amplios de conversación (e.g., 20 a 30 turnos de palabra) que preceden y culminan en un MC.

Desde la exploración de MC y EC, Krause y colegas (e.g., Krause, 2005; Fernández et al., 2015) han desarrollado la teoría del cambio subjetivo (TCS). Conceptualizan el resultado o cambio terapéutico como una modificación en patrones subjetivos de interpretación y explicación, que llevan a los consultantes al desarrollo de nuevas teorías comprensivas de sí mismos (Fernández et al., 2015). Por su parte, el proceso de cambio — los MC y EC - se identifican de acuerdo a una jerarquía de 19 indicadores genéricos de cambio (IGC; Krause, 2005). Como muestra la Figura 1, el proceso se inicia con MC en los que se observa la aceptación de la existencia de un problema (IGC \#1), y culmina con MC que exhiben la construcción de una teoría subjetiva del cambio (IGC \#19).

\begin{tabular}{|c|c|}
\hline \multirow{5}{*}{$\begin{array}{l}\text { IGC } \\
\text { Nivel } 3\end{array}$} & 19. Construcción de una Teoria Subjetiva \\
\hline & 18. Disminución de la asimetría \\
\hline & 17. Reconocimiento de la ayuda recibida \\
\hline & 16. Autonomía de manejo del contexto de significado psicológico \\
\hline & 15. Enraizamiento de los constructos subjetivos en la propia biografía \\
\hline \multirow{5}{*}{$\begin{array}{l}\text { IGC } \\
\text { Nivel } 2\end{array}$} & 14. Formación de constructos subjetivos respecto de sí mismo \\
\hline & 13. Transformación de valoraciones y emociones en relación a sí mismo u otros \\
\hline & 12. Reconceptualización de los propios problemas y/o sintomas \\
\hline & 11. Establecimiento de nuevas relaciones \\
\hline & 10. Aparición de sentimientos de competencia \\
\hline & 9. Manifestación de un comportamiento nuevo \\
\hline & 8. Descubrimiento de nuevos aspectos de sí mismo \\
\hline & 7. Reconocimiento de la propia participación en los "problemas" \\
\hline & 6. Expresión de la necesidad de cambio \\
\hline & 5. Descongelamiento de formas habituales \\
\hline IGC & 4. Expresión de esperanza \\
\hline Nivel 1 & 3. Aceptación del terapeuta como profesional competente \\
\hline & 2. Reconocimiento de la necesidad de ayuda \\
\hline & 1. Aceptación de la existencia de un problema \\
\hline
\end{tabular}

Figura 1. 19 Indicadores de Cambio Genérico según la Teoría Subjetiva del Cambio. Los indicadores de proceso corresponden al Nivel 2. 
La Figura 1 muestra que los 19 IGC se agrupan en indicadores de entrada, proceso, y salida (nivel 1, 2, y 3; Fernández et al., 2012). Los IGC de entrada, nivel 1, (\#1 al \#7) se entienden como condiciones previas necesarias pero no suficientes para que se dé un proceso terapéutico. Un ejemplo es la aceptación del terapeuta como profesional competente (IGC \#3). Los IGC de salida, nivel 3, (\#14 al \#19) evidencian resultados terapéuticos más estables, marcan la proximidad del fin del proceso, que culmina con la construcción de una teoría subjetiva del cambio (IGC \#19).

El presente trabajo se focalizó en IGC de proceso o nivel 2 (\#8-\#13) que se ubican entre los IGC de entrada y salida. En el nivel 2 los indicadores marcan cambios iniciales transitorios, que de consolidarse contribuirían también a la generación de una teoría del cambio subjetivo en el nivel 3. Un ejemplo de IGC de nivel 2 es el descubrimiento de aspectos del sí mismo (\#8). En otras palabras, la teoría propone que segmentos conversacionales relativamente amplios (EC), que culminan en el logro de eventos de cambio (MC), que presentan este tipo de IGC de proceso (nivel 2), facilitan procesualmente el logro de indicadores de cambio de salida (nivel 3). De allí la importancia de comprender mejor los EC de nivel 2, es decir, especificar el tipo de micro-prácticas conversacionales que contribuyen a generar MC con indicadores de nivel 2.

Se exploraron los $16 \mathrm{EC}$ de proceso que fueron identificados en las primeras 18 sesiones de una terapia de orientación gestáltica exitosa. Haciendo un "zoom” en los EC, caracterizamos 6 micro-prácticas conversacionales que facilitaron el logro de dichos EC de proceso. Esta caracterización contribuye a la especificación de la teoría TCS, y en este sentido es un estudio de caso constructor de teoría (Stiles, 2007).

\section{Autorreferencias Preferidas como "Logro" Conversacional}

La TCS propone que en los EC se "logran" conversacionalmente momentos de cambio (MC). Estos logros implican transformaciones progresivas en cómo el consultante se reconoce, refiere y comprende a sí mismo en el transcurso de la conversación. La elaboración progresiva de estas autorreferencias culmina en la generación de una teoría subjetiva acerca del cambio (Arístegui et al., 2009).

La teoría es "subjetiva" ya que es validada por el consultante. Por otro lado, es una teoría "inter-subjetiva", ya que es generada (conversacionalmente) junto al terapeuta, quien valida - y así co-instituye - la teoría. Así, la TCS es coherente con la tradición dialógico-hermenéutica en psicología (e.g., Arístegui et al., 2009; Mead, 1934), según la cual el modo en que alguien ("yo") se autorrefiere ("mí") es el resultado de una internalización de la interacción/ conversación con "otros significativos" que reconocen y validan la autorreferencia.

En este estudio llamamos autorreferencias preferidas (ARP) a las caracterizaciones/evaluaciones del consultante ("mí"), aceptables para el consultante ("yo"), y validadas por el terapeuta ("otro significativo"), que se generan en los EC de proceso (nivel 2). Un supuesto es que las autorreferencias preferidas, al ser validadas intersubjetivamente, tienen más probabilidad de ser internalizadas, influyendo en la narración o "teoría subjetiva" relativamente dominante que el consultante produce sobre sí mismo y su proceso de cambio (IGC \#19). En función de la aceptación de estas evaluaciones-de-sí, una persona progresivamente clarifica para sí misma "quién es" (autodiálogo yo/mí). En otras palabras, en el proceso terapéutico el consultante progresivamente clarifica para sí su identidad al intentar clarificarla para otro (terapeuta). En el tiempo, la teoría sobre "mí" no solo representa o refleja cómo el "yo" experimenta el mundo, sino también lo prefigura o moldea — lo que teóricamente ayuda a mantener el cambio (Arístegui et al., 2009).

El presente estudio de procesos de cambio examina una expectativa central de la TCS: que en los episodios de cambio de proceso (EC de nivel 2) debieran observarse autorreferencias preferidas que son logradas por cliente y terapeuta a través del diálogo. La pregunta guía de este estudio apunta a especificar qué micro-prácticas conversacionales contribuyen al logro de autorreferencias preferidas (ARP) en este nivel 2. 


\section{Método}

El diseño del estudio fue exploratorio cualitativo, orientado a caracterizar micro-prácticas conversacionales que contribuyen a la generación de ARP de nivel 2. Inspirada en la tradición etnometodológica (Heritage, 1984; Wooffitt, 2005), la exploración busca distinguir micro-prácticas o "métodos" conversacionales que los propios participantes (terapeuta y consultante) ocupan para conseguir cambio terapéutico, operacionalizado como MC de nivel 2. Para realizar un análisis del discurso en profundidad, se seleccionó un proceso terapéutico exitoso, que presentara suficientes EC para encontrar variabilidad discursiva (Wooffitt, 2005).

\section{Participantes y Procedimientos de Recolección de Datos}

Se consideró para el análisis un proceso terapéutico individual, exitoso, de orientación guestáltica, con un total de 61 sesiones videograbadas. La consultante es una mujer de 33 años de edad, soltera, con pareja homosexual. El terapeuta un hombre de 45 años de edad, de formación gestáltica, con 20 años de experiencia profesional.

El proceso terapéutico analizado se seleccionó a partir de una base de datos que incluye 14 procesos psicoterapéuticos completos, en el contexto de la implementación de dos proyectos financiados por el gobierno chileno a través del Fondo Nacional de Ciencia y Tecnología (FONDECYT, proyecto $\mathrm{N}^{\mathrm{o}}$ 1060768). Se seleccionó de acuerdo a un criterio de éxito terapéutico (de acuerdo a los criterios de éxito terapéutico del cuestionario OQ-45.2) y un criterio de variabilidad discursiva (Wooffitt, 2005). Es decir, un caso exitoso y suficientemente rico en episodios de cambio EC, como para permitir un análisis discursivo exploratorio de prácticas conversacionales (que contribuyeran al logro de aquellos EC). Se estimó que para encontrar variabilidad discursiva, se requerían explorar entre 15 y $20 \mathrm{EC}$ de nivel 2, ocurridos en un mismo proceso terapéutico. Los criterios de selección de MC, EC, y clasificación de IGC fueron los mismos de otras investigaciones similares (e.g., Fernández et al., 2015; Krause et al., 2006).

Investigadores independientes (Krause et al., 2006), entrenados en la identificación de MC y EC, codificaron las primeras 18 sesiones del proceso terapéutico, identificando un total de 38 EC. La codificación se realizó mediante la observación del proceso psicoterapéutico por parte de dos jueces entrenados, quienes seleccionaron independientemente los MC/EC, clasificándolos de acuerdo a los 19 ICG según el Manual de Observación y Codificación de los Episodios de Cambio y Estancamiento (Fernández et al., 2015). Se seleccionaron sólo los $38 \mathrm{EC}$ que fueron identificados por ambos jueces. De los $38 \mathrm{EC}$ los autores del presente estudio seleccionaron un total de 16 episodios (correspondientes a los EC que los jueces identificaron como conteniendo indicadores de cambio de proceso o IGC de nivel 2).

\section{Análisis de datos}

Para este estudio los $16 \mathrm{EC}$ de proceso fueron analizados en dos momentos:

Identificación de autorreferencias preferidas. Se exploró qué enunciados fueron tratados por los participantes como "evaluaciones." Para ello, se analizó la secuencia de acciones básicas (i.e., en términos de pares adyacentes; Wooffitt, 2005). Por ejemplo, un enunciado se consideró como una "pregunta" (acción básica), sólo si el interlocutor la trata como tal (al ofrecer una respuesta, se forma el par adyacente pregunta-respuesta). Se consideró como "evaluación" sólo aquellos enunciados cuyo interlocutor interpretó como tal (al aceptar, o al rechazar).

Posteriormente se interpretó qué evaluaciones fueron tratadas por los participantes como ARP (i.e., como implicando una caracterización / evaluación aceptable de algún aspecto relativo al consultante). Por ejemplo, en el siguiente extracto, la consultante $(\mathrm{C})$ proyecta una evaluación al posicionarse como "distinta" (línea 12). El terapeuta $(\mathrm{T})$ nota y acepta parcialmente la evaluación en la línea 14 (línea 14; la aceptación definitiva ocurre en el MC, al final del episodio). En efecto, el turno de T proyecta un pedido de que $\mathrm{C}$ especifique su autoevaluación (pedido que $\mathrm{C}$ reconoce como tal en la línea 15). 
Tabla 1. Extracto de diálogo entre consultante (C) y terapeuta (T).

\begin{tabular}{|l|l|l|}
\hline Línea & Hablante & Diálogo \\
\hline 11 & $\mathrm{C}$ & siento que he empezado a verme desde hace qué sé yo, hace una algo así \\
\hline 12 & & como un mes, por primera vez de una manera distinta a como me sentí durante unos \\
\hline 13 & & quince años, o una cosa así \\
\hline 14 & T & cómo te estás viendo en este último tiempo? \\
\hline 15 & C & me estoy viendo más abierta \\
\hline
\end{tabular}

Caracterización de micro-prácticas conversacionales. Las acciones "básicas" previamente identificadas (autoevaluaciones o ARP) se interpretaron como generadas por acciones de rango medio mediadas institucionalmente. Como sugiere Antaki (2008), estas acciones son maneras de conversar que se consideran institucionalmente idiosincráticas, en el sentido de que realizan un "trabajo institucional" (p. 34), como es el de generar cambio terapéutico. En el extracto anterior, por ejemplo, se interpretó que la ARP identificada ("distinta a como me sentí...") era parte de una micro-práctica conversacional de rango medio que describimos como Notar. En esta práctica, la díada logra validar intersubjetivamente que el consultante ha comenzado a "notar" un cambio positivo. Aun cuando no pueda consensuar en qué específicamente consiste dicho cambio, este notar cambio supone una forma de autorreferencia, ya que es relativo al sí mismo de la consultante. Consideramos este modo de conversar como realizando un trabajo institucional (en tanto que ayudó procesualmente a la generación de cambio terapéutico, operacionalizado en la ocurrencia de un MC conteniendo un IGC de nivel 2: "manifestación de un comportamiento nuevo"). Así, la práctica de Notar permitiría que terapeuta y consultante coordinen un foco autorreferencial inicial, sobre el que continuarán elaborando el resto del EC, hasta concluir en un MC clasificado como IGC \#9 (En la siguiente sección de "Resultados" describimos en detalle cada una de las seis micro-prácticas conversacionales encontradas).

\section{Resultados}

Distinguimos seis prácticas a través de las cuales los participantes lograron discursivamente generar y acordar autorreferencias: notar, simbolizar, agencializar, apreciar, justificar, y ejemplificar. La mayoría de estas prácticas aparece en los $16 \mathrm{EC}$ analizados, y en forma concatenada. Es decir, si bien no todas las prácticas aparecen en todos los EC, en la mayoría la díada terapéutica primero logra (1) notar un foco de trabajo, y luego lo (2) simboliza; o primero lo simboliza y luego lo (3) agencializa y/o (4) aprecia; o primero lo aprecia y luego lo (5) justifica y/o (6) sitúa, etc. Dado el carácter exploratorio de este estudio, sin embargo, no se consideró ni la frecuencia ni la concatenación como un elemento relevante para el reporte. Por motivos de espacio, y para evitar tener que dar contexto a cada EC, ilustraremos la mayoría de las prácticas utilizando los dos primeros EC (episodios \#1 y \#2, que aparecieron en la sesión $\mathrm{N}^{\circ} 4$ de la terapia analizada). Excepcionalmente utilizaremos otros dos EC (\#16 y \#19, de las sesiones $\mathrm{N}^{\circ} 14$ y 18 respectivamente), para ilustrar prácticas que no se aprecian suficientemente en los dos primeros EC.

\section{Notar un foco}

A través de esta práctica conversacional, consultante y terapeuta logran una autorreferencia al distinguir, hacer público, y atender conjuntamente a un foco de atención relativo al "yo" de la consultante: una diferencia relevante en cómo ella se percibe a sí misma. La díada terapéutica constata (i.e., consensua, valida y co-instituye) que se ha producido un cierto cambio significativo para el proceso terapéutico; un cambio aún no especificado en su contenido, pero que da cuenta de la percepción de una diferencia significativa relativa al sí mismo del consultante.

Esta práctica se observa en el primer episodio de cambio de todo el proceso (EC \#1), registrado en la cuarta sesión de la terapia analizada. El terapeuta $(\mathrm{T})$ invita a la consultante $(\mathrm{C})$ a repetir un ejercicio de sillas calientes, 
que es común en la terapia de orientación gestáltica. T le pide a C que actúe un diálogo entre dos "partes" de C: (a) la parte "racional, controladora" en contraposición a (b) la parte del "monstruito afectivo." El inicio del EC \#1 coincide con el inicio del ejercicio, luego de que $\mathrm{T}$ le pide a C-racional que le cuenta a C-afectiva "cómo te ves a ti y a ella en este momento de la vida de ella":

Tabla 2. EC\#1, Sesión 4 (extracto). Los silencios mayores a 1 segundo se indican en paréntesis, en décimas de segundo.

\begin{tabular}{|l|l|l|}
\hline Línea & Hablante & Diálogo \\
\hline 1 & C & yo siento en el fondo como que la vida de las dos ha cambiado bastante \\
\hline 2 & T & Mhh \\
\hline 3 & C & como (6.0) no sé si es costumbre o familiarización pero en el fondo ya no me \\
\hline 4 & & parece- ¿le hablo a ella? \\
\hline 5 & T & sí, háblale háblale \\
\hline
\end{tabular}

En la línea 1, C proyecta una autoevaluación que es reconocida por T en el turno siguiente ("mhh"). A su vez, $\mathrm{C}$ trata la respuesta de $\mathrm{T}$ como aceptación lo suficientemente débil como para que $\mathrm{C}$ persista clarificando su evaluación. C tiene una partida falsa ("en el fondo ya no me parece- le hablo a ella?"), en la que invita a T a que éste le confirme que está realizando el ejercicio en forma apropiada (línea 5). Si bien en este extracto C no aclara en qué consiste la diferencia notada, nos parece que aquí la díada ya logra un micro-resultado conversacional relevante para el proceso. Lo que este reconocimiento conjunto logra es una autoevaluación (i.e., una autorreferencia) mediante la construcción de dos posiciones temporales de sí (C-racional antes/C-racional ahora), y reclama para sí una posición-ahora distinta a la anterior. T reconoce y acepta esta diferencia en la continuidad del sí mismo (Bamberg et al., 2011). Algo similar se observa unos 3 minutos más adelante en la sesión, en el EC \#2:

Tabla 3. EC\#2, Sesión 4 (extracto).

\begin{tabular}{|l|l|l|}
\hline Línea & Hablante & Diálogo \\
\hline 11 & C & siento que he empezado a verme desde hace qué sé yo, hace una algo así \\
\hline 12 & & como un mes, por primera vez de una manera distinta a como me sentí durante unos \\
\hline 13 & & quince años, o una cosa así \\
\hline 14 & T & ¿Cómo te estás viendo en este último tiempo? \\
\hline
\end{tabular}

La respuesta de $\mathrm{T}$ (línea 14) reconoce el turno anterior de C (líneas 11-13) como unidad significativa (autoevaluación), y proyecta una pregunta sobre el contenido del cambio notado. Es decir, más que problematizar si hubo o no hubo cambio, T trata como problemático — como requiriendo mayor clarificación - el contenido de dicho cambio. En los dos extractos (EC\#1 y EC \#2), la díada parece acordar que una diferencia relevante relativa al sí mismo de la consultante ha sido registrada-notada. Como mostramos en los siguientes dos apartados, tanto en EC\#1 como en EC\#2 estas autorreferencias fueron un micro-logro procesual importante (si bien no suficiente) para la generación de un momento de cambio al final de los EC \#1 y \#2. Si bien encontramos solo dos instancias de la práctica Notar, nos parece significativo que estas se encontraran al inicio del proceso de cambio en el caso observado (EC \#1 y EC \#2). El resto de las prácticas que describimos parecen haberse generado a partir de haber logrado este foco autorreferencial compartido. 


\section{Simbolizar un foco}

Se refiere al logro de autorreferencias a través de la formulación exitosa (i.e., aceptable intersubjetivamente) de términos semánticos para describir un foco. Es decir, se trata de la caracterización del "contenido" de una diferencia significativa (notada en el pasado, presente, o proyectada en el futuro; ver apartado anterior). El terapeuta $\mathrm{T}$ trata el foco autorreferencial como una especie de objeto describible. Así, invita a $\mathrm{C}$ a establecer discriminaciones cualitativas que permiten dar forma (simbólica) al foco autorreferencial. El siguiente extracto ilustra cómo la díada P-T continuó en el EC \#1 simbolizando luego de haber notado una diferencia relevante:

Tabla 4. EC\#1. Sesión 4 (extracto). Los silencios mayores a 1 segundo se indican en paréntesis, en décimas de segundo.

\begin{tabular}{|l|l|l|}
\hline Línea & Hablante & Diálogo \\
\hline 1 & C & yo siento en el fondo como que la vida de las dos ha cambiado bastante \\
\hline 2 & T & Mhh \\
\hline 3 & C & como (6.0) no sé si es costumbre o o familiarización pero en el fondo ya no me \\
\hline 4 & & parece- le hablo a ella? \\
\hline 5 & T & si, háblale háblale \\
\hline 6 & C & sí, no, no, ya no me parece ni tan chico ni tan bonito ni tan volcánico ni tan \\
\hline 7 & & apretado, así como (3.0) me parece un poco más accesible, yo siento que hemos \\
\hline 8 & & avanzado en eso \\
\hline 9 & T & en qué sentido, cuéntame en qué sentido este- \\
\hline 10 & C & más así como más blandita a estar como más blandita, más ehh permeable \\
\hline 11 & T & aceptando que lleguen más a ella, una cosa así \\
\hline
\end{tabular}

En las líneas 6-7, C formula términos semánticos ("ni tan chico...más accesible"), y proyecta una nueva evaluación ("hemos avanzado en eso"), que T trata como requiriendo clarificación en la línea 9. En su respuesta, C formula una auto-descripción ("más así como más blandita...permeable"), cuyo reconocimiento (vía reformulación en línea 11; Bavelas et al., 2000) la constituye como autorreferencia exitosa. Algo similar ocurre en la continuación del EC \#2:

Tabla 5. EC\#2. Sesión 4 (extracto). Los silencios mayores a 1 segundo se indican en paréntesis, en décimas de segundo.

\begin{tabular}{|l|l|l|}
\hline Línea & Hablante & Diálogo \\
\hline 11 & P & siento que he empezado a verme desde hace que sé yo hace una algo así \\
\hline 12 & & como un mes, por primera vez de una manera distinta a como me sentí durante unos \\
\hline 13 & & quince años o una cosa así \\
\hline 14 & T & ¿Como te estás viendo en este último tiempo? \\
\hline 15 & P & me estoy viendo más abierta me veo más grande me veo más \\
\hline 16 & & también, más flexible más blandita más así como que como que me asomo como que \\
\hline 17 & & tengo algún atisbo de movimiento, como que ya no- no es una cosa así muy \\
\hline 18 & & acurrucada y muy cerrada \\
\hline 20 & P & mucha vergüenza, que pasó con esa vergüenza \\
\hline
\end{tabular}


En la línea 14, T pide una elaboración de la evaluación proyectada por $\mathrm{C}$ en el turno anterior. Como respuesta, $\mathrm{C}$ propone una serie de discriminaciones cualitativas ("más abierta...más grande...más flexible...más blandita.....atisbo de movimiento... [ya no] acurrucada...cerrada.”). La validación (también vía re-formulación) de $\mathrm{T}$ en la línea 19, y revalidación de C en la línea 20 la constituyen como una nueva autorreferencia exitosa.

En todas las instancias que codificamos como simbolización, los participantes exhibieron un uso expresivo del lenguaje, en el sentido de que los términos que se aceptan no son un mero signo que denota o refiere a otra cosa (ver e.g., palabra-símbolo; Gadamer, 2004). Los participantes coordinan un contenido particular para presentar una autorreferencia "como-algo." Dado que a través de esta práctica los participantes logran dar forma simbólica a lo que "le pasa" al consultante (i.e., al sí-mismo "narrado"), podría decirse que esta práctica contribuye a especificar el "mí." (Mead, 1934).

A diferencia de la práctica notar, cabe señalar que la práctica que llamamos simbolización fue bastante generalizada (estuvo presente en 14 de los 16 EC analizados). Si bien la presencia de estas dos prácticas contribuyó a la generación discursiva de un MC (observado hacia el final de cada EC), en ningún EC la presencia de estas dos prácticas fue suficiente para dicho logro conversacional.

\section{Agencializar un foco}

A través de esta práctica los participantes tratan el foco autorreferencial como algo que el consultante hizo, hace, o podría hacer. Es decir, las verbalizaciones utilizadas construyen al consultante como-alguien: como agente o entidad/persona con capacidad de acción (e.g., "ahora no dejas que te pase eso"; "ahora puedo"). En este sentido, incluimos también dentro de esta categoría aquellas prácticas conversacionales en las que la díada logró una autorreferencia al aceptar descripciones agenciales de "partes" de sí (e.g., nominalizaciones como "la racional", "la controladora", o "el monstruito"), cuando la díada se orienta a ella como algo que el consultante hace, o como una posición-yo (Arístegui et al., 2009). El siguiente extracto muestra cómo T continuó facilitando la autodescripción de la parte "racional" en el EC\#1, a través de la práctica de agencializar:

Tabla 6. EC\#1. Sesión 4 (extracto). Los silencios mayores a 1 segundo se indican en paréntesis, en décimas de segundo.

\begin{tabular}{|l|l|l|}
\hline Línea & Hablante & Diálogo \\
\hline 6 & C & sí, no no ya no me parece ni tan chico ni tan bonito ni tan volcánico ni tan \\
\hline 7 & & apretado, así como (3.0) me parece un poco más accesible, yo siento que hemos \\
\hline 8 & & avanzado en eso \\
\hline 9 & T & en qué sentido cuéntame en qué sentido este \\
\hline 10 & C & más así como más blandita a estar como más blandita más ehh permeable \\
\hline 11 & T & aceptando que lleguen más a ella una cosa así \\
\hline 12 & C & exactamente aceptando sacando algunas cosas, siento que ha sido súper positivo \\
\hline
\end{tabular}

Entendemos que la reformulación de $\mathrm{T}$ en la línea 11 hace algo más que meramente aceptar y reflejar (Cormier, Nurius \& Osborn, 2016) la formulación de C (línea 10). Según Antaki (2008, p. 34), los psicoterapeutas presentan sus (re)formulaciones como si fueran un "mero resumen neutral o implicación" de lo dicho por los consultantes, a la vez que aprovechan la oportunidad para "editar" lo dicho por ellos como forma de optimizar la agenda institucional (i.e., el cambio terapéutico). En este caso, interpretamos que en su formulación (línea 11) T posiciona discursivamente a una "parte" de C no sólo como su como sujeto de una experiencia (e.g., sentirse "más blandita"), sino como agente involucrado en la producción de dicha experiencia - como alguien que hace algo ("aceptando que lleguen a ella"). C confirma la propuesta de $\mathrm{T}$ en la línea 12 , y con ello la díada logra un nuevo tipo de autorreferencia. Algo similar ocurre en el proceso conversacional observado en el EC \#2: 
Tabla 7. EC\#2. Sesión 4 (extracto). Los silencios mayores a 1 segundo se indican en paréntesis, en décimas de segundo.

\begin{tabular}{|l|l|l|}
\hline Línea & Hablante & Diálogo \\
\hline 15 & C & me estoy viendo más abierta me veo más grande $\{$ T: incomprensible\} me veo más \\
\hline 16 & & también, más flexible más blandita más así como que como que me asomo como que \\
\hline 17 & & tengo algún atisbo de movimiento, como que ya no- no es una cosa así muy \\
\hline 18 & & acurrucada y muy cerrada \\
\hline 19 & T & mucha vergüenza, que pasó con esa vergüenza \\
\hline 20 & C & eh siento que está ahí, que está como a la mano y no quiero tomarla \\
\hline 21 & T & Mmhhh \\
\hline 22 & C & pero que está, que es tentadora $\{$ T: mhh $\}$, así como es tentadora porque es conocida \\
\hline 23 & & y esto es desconocido y si bien ha sido bueno para mí ha sido entretenido así como \\
\hline 24 & & salir gustarme hacer cosas distintas disfrutar un poco \\
\hline 25 & T & qué cosas has hecho tú por ejemplo, que cosas sientes tú que en donde te has \\
\hline 26 & & mostrado concretamente así como en qué lugar en que momento \\
\hline
\end{tabular}

En la línea 20, C se orienta al pedido de clarificación de T (línea 19) reclamando para sí una posición agencial ("no quiero tomarla"), que es validada por T en el turno siguiente (nótese que el "Mmhhh" fue realizado con mayor énfasis en comparación a otras muestras de reconocimiento o acknowledgment tokens; Wooffitt, 2005). En las líneas 22-26 C continúa posicionándose como agente que responde frente a su experiencia simbolizada/objetivada ("esa vergüenza"). Un agente que se resiste (a dejarse claudicar por la vergüenza tentadora), elige (lo "desconocido"), y hace "cosas distintas." En el turno siguiente, C reconoce y valida este posicionamiento agencial.

Compárese con lo observado al inicio el último EC analizado (\#19, sesión \#18), ocurrido a continuación de que $\mathrm{T}$ invita a $\mathrm{C}$ a evaluar lo que ella "rescata" del proceso terapéutico. La díada comienza a agencializar después de la línea 16. Previamente (líneas 1-16) la díada parece simbolizar la experiencia de P (i.e., nominalizan la experiencia de P como-algo: "esa seguridad"; "la angustia"; "el tema de los límites", "el tema del lado oscuro"). A partir de la línea 16, sin embargo, sus autorreferencias tratan a $\mathrm{C}$ como un sujeto o agente: como-alguien que prioriza (línea 16), y que hace apostolados (línea 20): 
Tabla 8. EC\#19. Sesión 18 (extracto). Los silencios se indican en paréntesis. Las respuestas que se superponen se indican con " \{\} ". Volumen bajo se indica entre "o o".

\begin{tabular}{|c|c|c|}
\hline Línea & Hablante & Diálogo \\
\hline 1 & T: & claro como que está esa seguridad pero no hay seguridad de que se mantenga \\
\hline 2 & P: & claro, claro ${ }^{\circ}\{\mathrm{T} \text { : es de esperar }\}^{\circ}$ y no es que este ahora puntualmente \\
\hline 3 & & sino que ha estado en determinados momentos \\
\hline 4 & $\mathrm{~T}:$ & como un poco azarosa sin que tú la manejes mucho \\
\hline 5 & P: & claro así como que como que se turnan un poco $\{\mathrm{T}: \mathrm{hmm}\}$ como con la \\
\hline 6 & & angustia $\{\mathrm{T}: \mathrm{hmm}\}$ entonces yo creo que eso ha sido como lo más \\
\hline 7 & & rescatable el tema como de ir clarificando que tiene:: eh un montón de \\
\hline 8 & & consecuencias un montón de secuelas: como que disminuya la \\
\hline 9 & & angustia como que en determinado momentos haya \\
\hline 10 & T: & no estás tan perdida en un caos (inaudible) digamos \\
\hline 11 & P: & claro claro el tema de los límites ha sido súper claro también el tema \\
\hline 12 & & como de la como del lado oscuro digamos lo tengo como bien claro \\
\hline 13 & & también, no sé si comparto el:: eh como desde la teoría: lo que significa \\
\hline 14 & & pero más allá de eso eh (3.0) claro lo puedo mirar desde acá o lo \\
\hline 15 & & puedo mirar desde acá y en el fondo sigue siendo el mismo paquete \\
\hline 16 & & $\{\mathrm{~T}: \mathrm{hmm}\}$ entiendo este tema de priorizar al resto por sobre:: por sobre \\
\hline 17 & & mis necesidades ${ }^{\circ}\{\mathrm{T}: \mathrm{hmm}\}^{\circ}$ por sobre: mis ganas por sobre mi querer \\
\hline 18 & & incluso por sobre mi seguridad digamos ${ }^{\circ}\{\mathrm{T}: \mathrm{hmm}\}^{\circ}$ así como $\mathrm{mm} ?$ \\
\hline 19 & & entonces es casi como un:: no sé cómo se dice pero como un \\
\hline 20 & & apostolado así como que $\{\mathrm{T}$ se ríe $\}$ yo me voy a angustiar y me voy a \\
\hline 21 & & insegurizar para que tu estés bien \\
\hline 22 & T: & esas son cosas también que se han ido clarificando en el trayecto \\
\hline 23 & P: & claro, claro \\
\hline
\end{tabular}

La práctica agencializar fue identificada en los $16 \mathrm{EC}$ examinados. En todos los casos, más que significar el foco como-algo que a la persona le pasa ("mí"), terapeuta y consultante se orientan a así un agente responsable de la acción; como-alguien ("yo") que responde a una circunstancia (e.g., que acepta que lleguen a ella; que se muestra; que prioriza al resto).

\section{Apreciar o tomar una posición}

Mediante esta manera de conversar, el terapeuta y la consultante consiguen una autorreferencia al suponer (y clarificar) una toma de posición para el consultante. La díada construye un determinado curso de acción, modo de ser o posición como preferida. Por ejemplo, la díada continuó el diálogo de partes en EC\#1 construyendo la nueva posición-yo generada ("aceptando") como moralmente preferible. En efecto, este estar "aceptando" es tratado como moralmente preferible a la nominalizada parte racional/controladora (para quien todo lo que "sale" del "monstruito" es "como mierda"): 
Tabla 9. EC\#1. Sesión 4 (extracto). Los silencios se indican en paréntesis.

\begin{tabular}{|l|l|l|}
\hline Línea & Hablante & Diálogo \\
\hline 10 & $\mathrm{C}$ & más así como más blandita a estar como más blandita más ehh permeable \\
\hline 11 & $\mathrm{~T}$ & aceptando que lleguen más a ella una cosa así \\
\hline 12 & $\mathrm{C}$ & exactamente aceptando sacando algunas cosas, siento que ha sido súper positivo \\
\hline 13 & & las por lo menos las primeras cosas que han salido, entonces se, quedas como \\
\hline 14 & & desmitificado un poco este tema de de todo lo que sale es como mierda digamos ehh \\
\hline 15 & T & Hmm o sea es decir tu no solo la ves mejor sino que lo que ha salido de ella lo ves positivo \\
\hline 16 & C & lo veo positivo, claro y (3.0) \\
\hline
\end{tabular}

En las líneas 12-14 C construye a la parte desde la que habla (C-racional, "la controladora") como proyectando una evaluación sobre "el monstruito" (la parte a la que le habla en el ejercicio). Esta evaluación atribuida a "la controladora" es reconocida y formulada por T en la línea 15 (“...tu no sólo la ves mejor sino que lo que ha salido de ella lo ves positivo). En la línea 16, C confirma esta formulación, constituyendo la nueva posición-yo ("aceptando") como preferible. Si bien típicamente las apreciaciones que observamos fueron explícitas (e.g., "ha sido bueno... positivo"), encontramos instancias (EC \#7, \#16, \#18) en que las apreciaciones ocurrieron en forma indirecta. Por ejemplo, el EC\#16 (sesión 14) la díada clarifica la "necesidad" de cambiar un hábito, como un modo indirecto de apreciar o pronunciarse (i.e., tomar una posición) respecto de dicho hábito. En dicho episodio se registra un diálogo de partes entre (a) la destruida/cansada" y (b) la red bull/con mucha energía:

Tabla 10. EC\#16. Sesión 14 (extracto). Los silencios se indican en paréntesis. Las respuestas que se superponen se indican con " \{\} ".

\begin{tabular}{|l|l|l|}
\hline Línea & Hablante & Diálogo \\
\hline 8 & C & yo no puedo decir eso, entonces no sé, si desde esta postura, \\
\hline 9 & & con este cansancio, con esta sensación de destrucción puedo \\
\hline 10 & & llegar a acoplarme a esta otra persona que es, así como red bull \\
\hline 11 & T & claro, así con mucha sed, con mucha energía \\
\hline 12 & C & con mucha energía y con, además siento que puedo entender desde los dos lados \\
\hline 13 & & a la parte muy bien, en términos racionales lo entiendo muy bien \\
\hline 14 & T & hmm entiendes la parte de ella también \\
\hline 15 & C & sí, perfectamente, $\{$ T:hmm $\}$ así como de hecho, (4.0) muy probablemente \\
\hline 16 & & necesito desde aquí una reconstrucción $\{$ T:hmm $\}$ para poder seguir explorando después \\
\hline 17 & T: & porque esa parte te encanta, cierto? \\
\hline 18 & C: & porque eso me gusta, lo necesito porque es como la $=$ \\
\hline 19 & T: & no te imaginarías siendo así siempre, el resto de la vida \\
\hline 20 & C: & no, no \\
\hline
\end{tabular}

Mediante sus intervenciones en las línea 17 y 19 , el $\mathrm{T}$ terapeuta formula las autorreferencias expresadas por $\mathrm{P}$ (“necesito desde aquí una reconstrucción...”; “...eso me gusta, lo necesito...), en términos de una postura preferida. Por su parte, C valida intersubjetivamente las propuestas de T (línea 18, y más claramente en la línea 20). 


\section{Justificar la posición}

Otra práctica observada que generó ARP fue la suposición de que las posturas tomadas por C estarían justificadas. Es decir, C y T colaboran en clarificar "razones" (i.e., explicaciones validadas intersubjetivamente) por las cuales algún curso de acción cuenta como preferencia. Los participantes aquí invocaban algún repertorio de ideas que justificaría una toma de postura o apreciación. A través de esta práctica los participantes explicitan por qué una autorreferencia es normativamente preferida (es decir, que se está justificado o que hay "buenas razones" para preferirla), y con ello logran fortalecer la validez de una autorreferencia. Por ejemplo, en una ocasión la consultante justificó una toma de decisión invocando su "derecho" a no ser invadida por otros (EC \# 18). El terapeuta, al endosar esta justificación, validó la toma de postura, y con ello (constituyó) la autorreferencia como preferida. Otros ejemplos de ARP exitosas en la forma de justificaciones incluyen la apelación a la importancia de no apresurarse para enfrentar conflictos profundos (EC \#7); la importancia de reconocer o "no tapar" los propios sentimientos (EC \#6); o la importancia de "validar" lo que uno siente (EC \#8). En el episodio recientemente analizado (EC \#16), vimos cómo la díada consiguió primero apreciar que "es necesario descansar" (para la "red bull"). Poco después en el mismo EC \#16, la díada avanzó desde le apreciar a justificar una postura preferida para la "red bull" ("es necesario descansar") apelando a la importancia del "equilibrio":

Tabla 11. EC\#16. Sesión 14 (extracto). Las respuestas que se superponen se indican con "\{\}\}".

\begin{tabular}{|l|l|l|}
\hline Línea & Hablante & Diálogo \\
\hline 51 & T & porque si no, no puedes seguir viviendo la vida \\
\hline 52 & C & no, claro $\{$ T: te falta el cuerpo $\}$ mi fin es otro, claro, no sé si yo estoy buscando el \\
\hline 53 & & equilibrio, estoy buscando estar preparada para, es como que voy a dormir de \\
\hline 55 & & aquí hasta el treinta y uno, porque el treinta y uno no duermo nada, mis fines son \\
\hline 56 & & otros, yo sé, pero, pero, pero el momento actual es \\
\hline
\end{tabular}

Razones de espacio nos impiden poner el EC completo, como para entender mejor cómo $\mathrm{T}$ y $\mathrm{C}$ coordinaron el significado de "equilibrio" en esta interacción. Sin embargo, creemos que estas pocas líneas permiten observar que T y C aceptan "estoy buscando el equilibrio" como razón válida. Según lo observado, la práctica que distinguimos como justificar involucra la utilización de razones y patrones de conducta culturalmente reconocibles y aceptables para engrosar o sedimentar la preferencia de una postura. En este sentido, podrían considerarse como repertorios interpretativos (Wooffitt, 2005) que los participantes invocan para lograr fijar conversacionalmente autorreferencias preferidas que hagan una diferencia en el proceso de cambio.

\section{Situar la posición}

A través de esta práctica los participantes "sitúan" una postura preferida al identificar ejemplos concretos (i.e., historias) construidas como parte de la vida cotidiana del consultante. Los participantes logran así identificar ejemplos de situaciones (reales en el pasado o posibles en el futuro) que se tratan como evidencia de una postura previamente coordinada como preferida. Para ilustrar esta práctica, considérese cómo la díada continuó aproximándose a un momento de cambio en el EC \#2 referido con anterioridad: 
Tabla 12. EC\#2. Sesión 4 (extracto). Los silencios se indican en paréntesis. Las respuestas que se superponen se indican con " \{\} ". Aumento en inflexión de la voz se indica con "?".

\begin{tabular}{|c|c|c|}
\hline Línea & Hablante & Diálogo \\
\hline 25 & $\mathrm{~T}$ & que cosas has hecho tú por ejemplo, que cosas sientes tú que en dónde te has \\
\hline 26 & & mostrado concretamente así como en qué lugar en qué momento \\
\hline 27 & $\mathrm{C}$ & me he mostrado en el trabajo me mostrado en términos sociales también \\
\hline 28 & $\mathrm{~T}$ & dame un ejemplo \\
\hline 29 & $\mathrm{C}$ & he hablado de mi por ejemplo \\
\hline 30 & $\mathrm{~T}$ & Ah? \\
\hline 31 & $\mathrm{C}$ & y he hablado de ti \\
\hline 32 & $\mathrm{~T}$ & y has hablado tu o ha hablado ella de ti? \\
\hline 33 & $\mathrm{C}$ & entre las dos \\
\hline 34 & $\mathrm{~T}$ & entre las dos, mano a mano? \\
\hline 35 & $\mathrm{C}$ & eh mano a mano, sí \\
\hline 36 & $\mathrm{~T}$ & y que cosas han contado de ti entre las dos? \\
\hline 37 & $\mathrm{C}$ & ehh, más o menos lo mismo que estoy contando \{T: aha ya\} así como como que hay \\
\hline 38 & & un poco más de apertura como que es un conocimiento nuevo así como había cosas \\
\hline 39 & & dentro de esta roca volcánica y así como que tira humo, así como los indios $\{\mathrm{T}: \mathrm{mhh}\}$ \\
\hline 40 & & y que se ve, que claramente se ve $\{\mathrm{T}: \mathrm{mhh}\}$ que tal vez no son, no van a cambiar \\
\hline 41 & & radicalmente taan de un momento a otro digamos pero pero que la gente lo ve, así \\
\hline 42 & & como mira, hay alguna señal de humo ahí, y ha sido muy positivo hablarlo también, \\
\hline 43 & & así como he sentido que soy bastante autosuficiente, si es que eso pudiera tener muy \\
\hline 45 & & buen sentido digamos \\
\hline 46 & $\mathrm{~T}$ & Ya \\
\hline 47 & $\mathrm{C}$ & soy bastante como, me he sentido durante este mes como no dependiendo mucho de mi otra parte \\
\hline 48 & $\mathrm{~T}$ & ah ya, como que tu sola tienes recursos \\
\hline 49 & $\mathrm{C}$ & como que tengo recursos $\{\mathrm{T}: \mathrm{mhh}\}$ como que he tenido una acogida del otro lado \\
\hline 50 & $\mathrm{~T}$ & Perfecto \\
\hline
\end{tabular}

Como señalamos, en este extracto T invita a C a hablarle a "la racional" desde la posición "el monstruito." Luego de (1) notar una diferencia en esta postura, de (2) simbolizarla como "más abierta", de (3) agencializarla como no querer "tomar" [la vergüenza], y de (4) apreciar esta postura ("ha sido bueno para mí"), T invita a C a formular un ejemplo concreto en que se hubiese materializado dicha postura preferida (líneas 25, 26, 28). Esto abre espacio para que $\mathrm{C}$ genere un nuevo tipo de autorreferencia entre las líneas 27 y 41 , que hemos llamado "situar." Resulta interesante notar que en este episodio la díada pasó de apreciar (líneas 23-24) a situar (líneas 25-42), y que con posterioridad parecieron culminar con justificación (líneas 43-50). Hemos dejado las líneas 43-50, para apreciar cómo la díada coordina que una de las razones más importantes de por qué esta postura es preferida, es que parece encarnar un ideal culturalmente reconocible de independencia psicológica o de ser "autosuficiente". Sin perjuicio de que el justificar puede haber contribuido a la detección de un momento de cambio (MC) al final de este episodio, creemos que la práctica de situar (líneas 27-41) fue un elemento relevante en la generación conversacional del MC. (Por razones de espacio omitimos aquí la transcripción del MC, en el que P formula la diferencia notada como que el monstruito le ha permitido "tomar decisiones." Esta formulación final parece explicar el que este MC fuera identificado como conteniendo el IGC \#9 - la manifestación de un comportamiento nuevo). 


\section{Discusión}

A través de un análisis discursivo de episodios de cambio (EC) en el proceso terapéutico exitoso analizado, distinguimos seis micro-prácticas conversacionales que contribuyen conversacionalmente en la generación de momentos de cambio (MC) de nivel 2 (ver Fig. 1). Pueden considerarse que estas seis prácticas constituyen así tareas institucionales (Antaki, 2008), en tanto ayudan procesualmente al logro de indicadores genéricos de cambio (IGC) de nivel 2, y con ello al logro de IGC de nivel 3 (o de salida; Fernández et al., 2012).

En base a los resultados, el rol institucional del terapeuta durante los EC (como facilitador de estas tareas) del proceso terapéutico analizado, podría parafrasearse como organizado en base a seis supuestos (o etno-métodos; Heritage, 1984). Según lo observado, durante los EC el terapeuta supone o da por sentado en su forma de responder frente al consultante (1) que las diferencias o cambios que el consultante ha percibido y comparte con el terapeuta en ese momento de la sesión son relevantes para el proceso (notar); (2) que esas diferencias o cambios percibidos se pueden caracterizar lingüísticamente de modo más o menos adecuado o "correcto" (simbolizar); (3) que hay un agente (el consultante) que genera dichos cambios o acciones (agencializar); (4) que el agente evalúa o toma postura sobre dichas acciones como preferidas (apreciar); (5) que la toma de postura obedece a razones (justificar), y que emerge en el contexto de una (pequeña) historia de vida (situar). Más aún, durante un mismo EC, terapeuta y consultante suponen que "aquello" que se nota, que se simboliza, que se hace, que se aprecia, que se justifica y que se sitúa, se refieren a "lo mismo". Es decir, las seis prácticas comparten un mismo objeto intencional (al menos al interior de un mismo EC).

De acuerdo a la teoría del cambio subjetivo (Arístegui et al., 2009; Krause et al, 2006), la conversación en un EC es autorreferencial en el sentido de que el objeto intencional sobre el que se conversa en estos episodios es el sí mismo del consultante ("mí"). A la luz de esta teoría, las seis micro-prácticas identificadas serían modos de coordinar autorreferencias preferidas (ARP), es decir, seis modos de formular lingüísticamente aspectos del consultante ("mí"), que son aceptables para el consultante ("yo"), y validadas por el terapeuta en tanto que "otro significativo". Debido a que se refieren a "lo mismo", en un EC el proceso de autorreferirse a través de estas seis prácticas se encuentra interrelacionado holísticamente: primero se nota algo, luego se simboliza ese algo", luego se agencializa ese algo simbolizado, etc.

Si representamos la generación de ARP como una cuerda, diríamos que en un EC la cuerda se va haciendo más gruesa y visible a medida que se le añaden nuevas "fibras". Esta metáfora puede ser útil teóricamente, ya que ayudaría a explicar cómo y por qué el logro de indicadores genéricos de cambio (IGC) de nivel 2, ayuda procesualmente al logro de IGC de nivel 3 o de salida. En la medida de que se logran más tareas, la ARP es más fuerte ("gruesa"); eso aumentaría la probabilidad de que ese modo de autorreferirse (en el nivel 2) sea sustentable, persista en el tiempo ("resista"), y acabe por internalizarse. Dicha internalización sería expresada en IGC de salida (nivel 3), tales como la formación de constructos subjetivos respecto de sí mismo (IGC \#14), el enraizamiento de estos constructos en la propia biografía (IGC \#15) o, en definitiva, la construcción de una teoría subjetiva (IGC \#19).

En este sentido, creemos que la TCS (e.g., Krause et al., 2006) no sólo es coherente con, sino que puede fortalecerse explicativamente al comprenderse desde la tradición dialógico-hermenéutica en psicología (e.g., Arístegui et al., 2009; Garfinkel, 1956; Harré 2012; Mead, 1934; Vygotsky, 1966). Desde esta tradición teórica, se conceptualiza el sí-mismo (y con ello, sus "cambios") como un logro discursivo-social en permanente transformación (e.g., Mead, 1934; Arístegui et al., 2009). El sí-mismo aparece dinámicamente en y a través del reconocimiento y validación de otros significativos (Mead, 1934), mediada por el lenguaje (diálogo). Se propone así que el diálogo con otros (yo/otro) es constitutivo de un sí mismo en permanente generación. Progresivamente, el diálogo yo/otro se internaliza, modificando la auto-comprensión o "subjetividad" de una persona (i.e., se transforma en diálogo interno, o autodiálogo yo/mí; Arístegui et al., 2009).

Aplicado a este estudio, diríamos que en el proceso de cambio el consultante clarifica una teoría sobre sí-mismo como efecto colateral de un trabajo permanente por clarificar para el terapeuta como otro significativo. Desde esta tradición dialógico-hermenéutica, lo que motiva una respuesta tanto en el diálogo yo-otro y en el autodiálogo yo/mí, son autorreferencias. Las autorreferencias son interpretaciones significativas acerca-de-mí, o cargadas moralmente; Arístegui et al., 2009; Harré, 2012). Caracterizaciones, narrativas, emociones, u otros dispositivos culturalmente disponibles para referir a un sí-mismo, y que gatillan una respuesta de aceptación o rechazo pues están culturalmente cargadas de valor. Estas autorreferencias son aceptadas o rechazadas "en 
concordancia con estándares de 'preferencia' socialmente válidos" (Garfinkel, 1956, p. 420). Además del acto ilocutivo "neutro" propio de una aserción (referir), incluyen una dimensión evaluativa - son posiciones-yo dentro de un orden moral local (ver e.g., Harré, 2012). De ahí que propongamos las autorreferencias como evaluaciones-de-sí preferidas (no-preferidas cuando lo que invitan es el rechazo propio o de otro significativo).

Además de estas consecuencias teóricas, creemos que los resultados son potencialmente relevantes en términos clínico-prácticos, en el sentido que permitirían orientar a terapeutas para facilitar cambio terapéutico. Sensibilizarse frente a la potencial contribución que prácticas tales como notar, simbolizar, agencializar, apreciar, justificar y situar tengan en la co-creación de autorreferencias preferidas "gruesas" (que contribuyan en la generación/internalización de una teoría subjetiva). Dado el énfasis de estas prácticas en la co-creación de un agente que toma conciencia de su(s) posición(es) respecto de su situación en el mundo, nos parece que estas prácticas son consistentes teóricamente no sólo con el enfoque gestáltico/humanista propio de la terapia analizada, sino también con otros modelos terapéuticos tradicionales (psicodinámicos, cognitivo-conductuales, sistémicos, y posmodernos). El énfasis en "agencializar" el cambio es también consistente con modelos trans-teóricos inspirados en superar la desesperanza, y fomentar el empoderamiento facilitando que el cliente se vea como agente del problema y/o la solución (e.g., Krause, 2005; Prochaska, 2013; Wampold \& Imel, 2015).

Sin embargo, el hecho de que estas prácticas hayan sido extraídas de un solo proceso psicoterapéutico, llama a considerar estos resultados con cautela. Investigación futura sobre si y cómo estas micro-prácticas se observan en otros procesos terapéuticos exitosos, con otras orientaciones teóricas, con otras poblaciones, motivos de consulta, etc., permitirán aumentar la generalidad de estos hallazgos. Por ahora, nos parece justificado teóricamente, y tenemos evidencia empírica incipiente, de que el proceso terapéutico puede entenderse como un trabajo conversacional de clarificación constitutiva. Un proceso en que el consultante progresivamente clarifica para sí su identidad y sus cambios al intentar clarificarla para otro. Un proceso que no solo representa el sí mismo, sino que lo co-instituye; lo crea con el poder de la palabra.

\section{Referencias}

Antaki, C. (2008). Formulations in psychotherapy. In A. Perakyla, C. Antaki, S. Vehvilainen \& I. Leudar (Eds.), Conversation analysis and psychotherapy (pp. 26-42). Cambridge, UK: Cambridge University Press.

Arístegui, R., Gaete, J., Muñoz, G., Salazar, J. I., Vilches, O., Krause, M., Tomicic, A., \& Ramírez, I. (2009). Diálogos y autorreferencia: procesos de cambio en psicoterapia desde la perspectiva de los actos de habla. Revista latinoamericana de psicología, 41, $277-289$.

Bamberg, M., De Fina, A., \& Schiffrin, D. (2011). Discourse and identity construction. In S. Schwartz, K. Luyckx \& V. Vignoles (Eds.), Handbook of identity theory and research (pp. 177-199). New York, NY: Springer.

Bavelas, J., Coates, L., \& Johnson, T. (2000). Listeners as co-narrators. Journal of Personality and Social Psychology, 79, 941-952. doi: 10.1037/0022-3514.79.6.941

Cormier, S., Nurius, P. S., \& Osborn, C. J. (2016). Interviewing and change strategies for helpers. Boston, MA: Brooks Cole

Elliott, R. (2010). Psychotherapy change process research: Realizing the promise. Psychotherapy Research, 20, 123-135. doi: 10.1080/10503300903470743

Fernández, O., Herrera, P., Krause, M., Pérez, J. C., Valdés, N., Vilches, O., \& Tomicic, A. (2012). Episodios de cambio y estancamiento en psicoterapia: características de la comunicación verbal entre pacientes y terapeutas. Terapia psicológica, 30, 5-22.

Fernández, O., Pérez, C., Gloger, S., \& Krause, M. (2015). Importancia de los Cambios Iniciales en la Psicoterapia con Adolescentes. Terapia psicológica, 33, 247-255.

Gadamer, H. G. (2004). Truth and method. New York, NY: Continuum.

Garfinkel, H. (1956). Conditions of successful degradation ceremonies. American journal of sociology, 61(5), 420-424.

Harré, R. (2012). Positioning theory: Moral dimensions of social-sultural psychology. En J. Valsiner (ed.) The Oxford handbook of culture and psycholog (pp. 191-206). New York: OUP

Heritage, J. (1984). Garfinkel and ethnomethodology. Cambridge, MA: Polity Press.

Krause, M. (2005). Psicoterapia y cambio. Una mirada desde la subjetividad. Santiago de Chile: Ediciones Universidad Católica de Chile.

Krause, M., De la Parra, G., Arístegui, R., Tomicic, A., Dagnino, P., \& Echávarri, O. (2006). Indicadores de cambio genéricos en la investigación psicoterapéutica. Revista Latinoamericana de Psicología, 38, 299-325.

Mead, G.H. (1934). Mind, self, and society. Chicago, EE.UU: University of Chicago Press. 
Prochaska, J.O. (2013). Transtheoretical model of behavior change. En Encyclopedia of behavioral medicine (pp. 1997-2000). Nueva York, NY: Springer.

Stiles, W. B. (2007). Theory-building case studies of counselling and psychotherapy. Counselling and Psychotherapy Research, 7, $122-127$.

Wooffitt, R. (2005). Conversation analysis and discourse analysis: A comparative and critical introduction. London, UK: Sage.

Vygotsky, L. S. (1966). Development of higher mental functions. In A. N. Leontyev, A. R. Luria \& A. Smirnov (Eds.), Psychological Research in the USSR (pp. 11-46). Moscow: Progress Publishers.

Wampold, B.E., \& Imel, Z.E. (2015). The great psychotherapy debate: The evidence for what makes psychotherapy work. Nueva York, NY: Routledge. 